\title{
Surgical treatment of a giant aneurysm of the left anterior descending artery presenting with ischemia
}

Nikolaos Kosmas ${ }^{1}$, Ioannis Andreou ${ }^{1}$, Vasilios Kollias ${ }^{2}$, Konstantinos Stamoulis ${ }^{1}$, Dimitrios Vrachatis $\mathrm{A}^{1}$, and Spyridon Deftereos $\mathrm{G}^{1}$

${ }^{1}$ Panepistemiako Geniko Nosokomeio Attikon - B Kardiologike Klinike

${ }^{2}$ Panepistemiako Geniko Nosokomeio Attikon

November 19, 2021

\begin{abstract}
A 70-year-old man presented to our hospital with non-ST elevation myocardial infarction and severely depressed left ventricular systolic function. Coronary angiogram revealed a giant fusiform aneurysm of the proximal left anterior descending artery with significant stenoses immediately proximal and distal to it and a left circumflex chronic total occlusion. The patient was treated surgically, with ligation and bypass of the aneurysm using a radial artery graft and a vein graft to the first obtuse marginal branch.
\end{abstract}

Surgical treatment of a giant aneurysm of the left anterior descending artery presenting with ischemia

October 2021

Nikolaos Kosmas ${ }^{\mathrm{a}}$, Ioannis Andreou ${ }^{\mathrm{a}}$, Vasilios D Kollias ${ }^{\mathrm{b}}$, Konstantinos Stamoulis ${ }^{\mathrm{a}}$, Dimitrios A Vrachatis ${ }^{\mathrm{a}}$, Spyridon G Deftereos ${ }^{\mathrm{a}}$

Affiliation: ${ }^{a}$ Second Department of Cardiology and ${ }^{\mathrm{b}}$ Department of Cardiac Surgery, Attikon University Hospital, Athens, Greece

Number of figures: 1

Word count: 407 (text only)

\section{Running title: Giant left anterior descending aneurysm}

Financial disclosure: There is no conflict of interest to disclose for any author. All authors have approved the final article. This scientific research has not received funding.

Statement: This clinical and educational work is in compliance with our institution's ethical committee standards. Written informed consent was obtained from the patient.

Keywords: Coronary artery aneurysm, aneurysm exclusion, coronary CT angiography, acute coronary syndrome

Abstract: A 70-year-old man presented to our hospital with non-ST elevation myocardial infarction and severely depressed left ventricular systolic function. Coronary angiogram revealed a giant fusiform aneurysm of the proximal left anterior descending artery with significant stenoses immediately proximal and distal to 
it and a left circumflex chronic total occlusion. The patient was treated surgically, with ligation and bypass of the aneurysm using a radial artery graft and a vein graft to the first obtuse marginal branch.

\section{Corresponding author:}

Spyridon G. Deftereos, MD, PhD

Professor of Cardiology

Second Department of Cardiology

Attikon Hospital, National and Kapodistrian University of Athens

1 Rimini St, Chaidari, Greece

e-mail: spdeftereos@gmail.com

A 70-year-old man was admitted to our hospital with angina, elevated high sensitivity troponin and unremarkable electrocardiogram. His medical history included coronary artery disease (lateral myocardial infarction conservatively treated 37 years ago), peripheral artery disease (endovascular repair of an abdominal aortic aneurysm 11 years ago), hypertension, dyslipidemia, obesity, active smoking, chronic obstructive pulmonary disease, chronic kidney disease, and psoriatic arthritis.

Echocardiography showed severely depressed left ventricular ejection fraction (35\%) with inferior, posterior and lateral wall akinesis. Coronary angiogram revealed a giant, 30x $25 \mathrm{~mm}$ fusiform aneurysm of the proximal left anterior descending (LAD) with significant stenoses immediately proximal and distal to it, and a chronic total occlusion of the left circumflex. Computed tomography delineated the morphology of the aneurysm, as well as the presence of penetrating ulcers of the aortic arch and descending aorta. Of note, a supra-renal aneurysm of the abdominal aorta with a maximum diameter of $70 \mathrm{~mm}$ was also observed.

The Heart Team decision for a surgical management of the patient was based on the proximal location and size of the aneurysm, the presence of significant coronary artery disease, and the impaired left ventricular systolic function. A radial artery graft was utilized to bypass the aneurysm, which was surgically excluded with proximal and distal ligation of the LAD. A saphenous vein graft was anastomosed to the first obtuse marginal branch. The left internal mammary artery was not employed taking into account patient's chronic obstructive pulmonary disease history and the planned thoraco-abdominal endovascular intervention with possible coverage of the left subclavian artery. The patient was discharged after a week in a good clinical condition.

Giant coronary aneurysms are usually clinically silent, but can present with ischemic symptoms or as an acute coronary syndrome (ACS) (1). Due to paucity of relevant randomized, controlled trials on the field, management of such patients is decided upon a case-by-case basis and can be medical (i.e., antiplatelet and/or anticoagulation regimens), percutaneous (i.e., using covered stents or stents plus coil embolization of the aneurysm), or surgical (2). The combination of a giant aneurysm with severe stenoses adjacent to it, presenting as an ACS is unusual. Surgery is considered first line treatment for proximally located giant aneurysms, while percutaneous treatment is often hindered by technical challenges, including availability of adequately sized stents, and higher failure rates (2). However, good results have been reported in selected cases with percutaneous interventions.

\section{References}

1. Núñez-Gil IJ., Cerrato E., Bollati M., et al. Coronary artery aneurysms, insights from the international coronary artery aneurysm registry (CAAR). Int J Cardiol 2020; 299:49-55.

2. Kawsara A., Núñez Gil IJ., Alqahtani F., Moreland J., Rihal CS., Alkhouli M. Management of Coronary Artery Aneurysms. J Am Coll Cardiol Intv 2018; 11(13):1211-23.

Figure and videos legend 
A: Coronary Angiogram cranial and B: caudal view.C, D and E: Computed tomography with 3D reconstruction. In the intraoperative views, the orientation is noted with human icon.F: Localization of the aneurysm. G: Proximal andH: distal ligation of the aneurysm. In all segments arrow shows the aneurysm. Star: proximal and distal to the aneurysm stenoses. Triangle: left circumflex total occlusion with retrograde filling via vollaterals. PV: pulmonary valve. LA: left auricle. U: ulcers of aorta. PA: pulmonary artery, Asc Aorta: ascending aorta. LM: left main coronary artery. LAD: left anterior descending coronary artery.

Video 1. Coronary angiogram (caudal view).

Video 2. Coronary angiogram (cranial view).

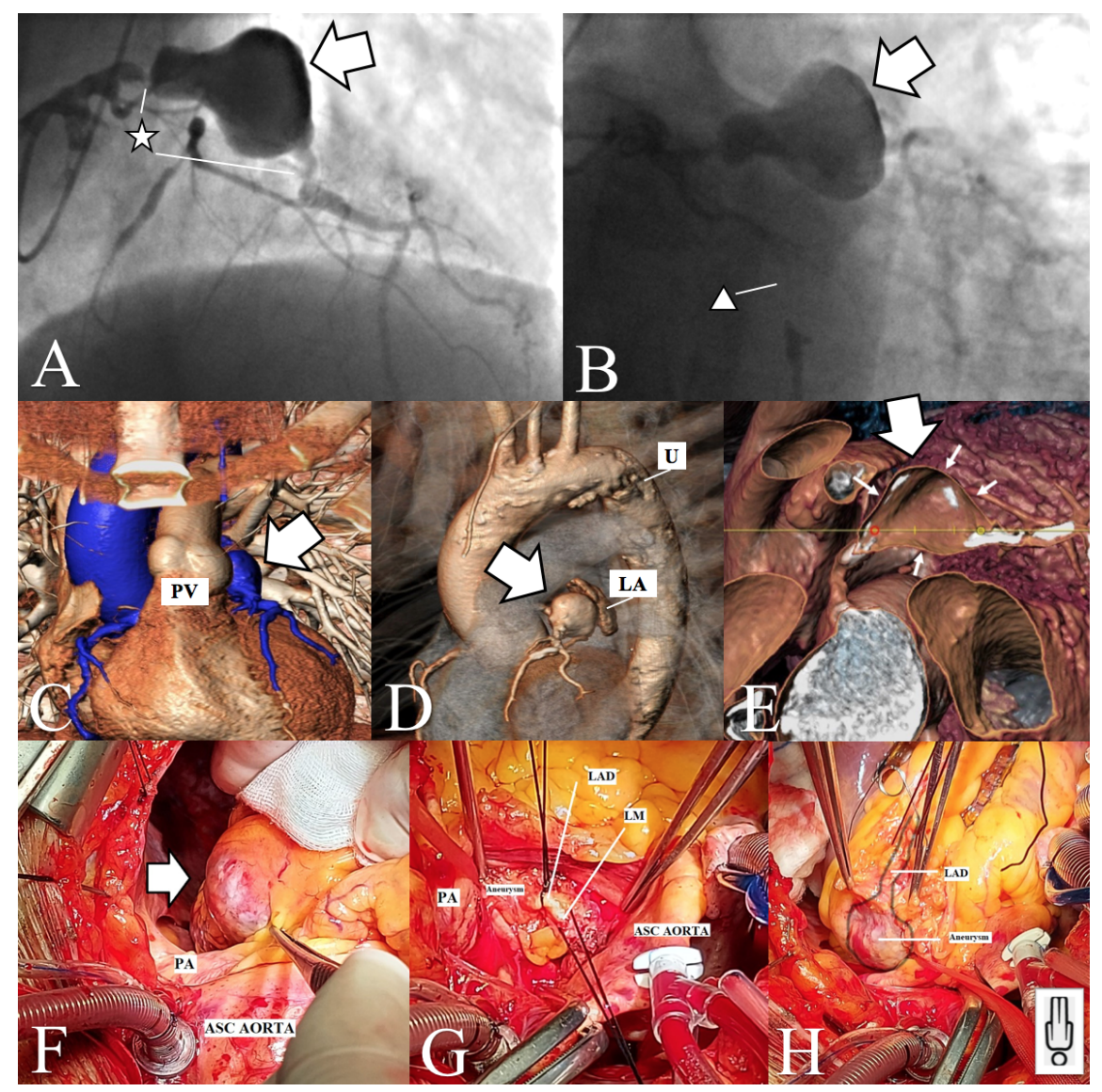

